NOVA

University of Newcastle Research Online

nova.newcastle.edu.au

Delgado, Ramón A.; Aguero, Juan C.; Goodwin, Graham C.; Yuz, Juan I. "Two-degree-offreedom anti-aliasing technique for wide-band networked control" Proceedings of the 18th IFAC World Congress (Milano, Italy ) p. 8884-8889 (2011)

Available from: $\underline{\text { http://dx.doi.org/10.3182/20110828-6-IT-1002.02710 }}$

Accessed from: http://hdl.handle.net/1959.13/1055715 


\title{
Two-degree-of-freedom anti-aliasing technique for wide-band networked control
}

\author{
Ramón A. Delgado* Juan C. Agüero* Graham C. Goodwin* \\ Juan I. Yuz ${ }^{* *}$ \\ * Centre for Complex Dynamics Systems and Control, The University \\ of Newcastle, Australia \\ ** Department of Electronic Engineering, Universidad Técnica \\ Federico Santa María, Chile
}

\begin{abstract}
In this paper we propose a new anti-aliasing technique for wide band networked control schemes. We study the potential equivalence with the usual one-degree-of-freedom antialiasing filtering. The benefits of the proposed anti-aliasing approach are illustrated with a simple example that incorporates an MPC controller in a Networked Control framework.
\end{abstract}

\section{INTRODUCTION}

Physical systems typically evolve continuously whereas modern controllers and signal processing devices invariably operate in discrete time (see e.g. Goodwin et al. (2010a,b)). Hence, it is necessary to have a device that transforms samples into continuous time signals (hold) and a device that takes samples of the relevant signals in the process to be controlled (see Figure 1). Conversion to sampled data form implicitly involves folding of high frequency system characteristics back into the lower frequency range. This effect is known as aliasing and means that different sampled signals may become indistinguishable. Aliasing is particularly problematic when wide-band noise is present. In order to avoid this problem an Anti aliasing filter (AAF) is used in analog to digital converters. The purpose of the AAF is to restrict the bandwidth of the signals such that the conditions in the sampling theorem are satisfied (Nyquist (1928)).

Recently, in Błachuta and Grygiel (2009a,b, 2010) the impact of the use of an AAF on the control loop performance is studied. It is argued, it is desirable to have an ideal $\mathrm{AAF}$ in order to avoid aliasing. However, an AAF also introduces a phase shift in the signal path (Goodwin et al. (2010b, 2001)). Thus, the performance and stability of the control loop are affected by incorporating an AAF.

In this paper, we study a new anti-aliasing filtering technique initially proposed in Goodwin et al. (2009, 2010b) called Two-Degree-of-Freedom Anti-aliasing filter (2-DOF-AAF). The proposed scheme is shown in Figure 3. This anti-aliasing filtering technique generalizes the usual One-Degree-of-Freedom Anti-aliasing filter (1-DOF-AAF). Choosing the filter $F_{1}=0$, we obtain the 1-DOF-AAF. Moreover, in the control loop in Figure 3, the controller can be modified such that, in some cases, both schemes are equivalent.

In this paper we show that the 2-DOF-AAF provides better performance than the 1-DOF-AAF. The loop (using 1-DOF-AAF) can even become unstable if a high order filter is used as an AAF due to the phase shift introduced in the loop. On the other hand, if a low order filter is used in the 1-DOF-AAF, then the impact of output disturbances with high frequency content is increased. In the 2-DOF-AAF architecture (Figure 3) one can be utilize an AAF of high order without affecting the closed loop performance. In addition, one can design the feedback law without taking into account the particular AAF utilised.

The layout of the paper is as follows: In Section 2, we present some technical preliminaries. In Section 3, we analyse the signals in the 2-DOF-AAF architecture. In Section 4, we study the potential equivalence between the 2-DOF-AAF and 1-DOF-AAF architectures. Section 5 shows the implication of the results in the context of Networked Control Systems (NCS). In section, 6 we present a simple example. Finally, we present conclusions in Section 7.

\section{TECHNICAL PRELIMINARIES}

In order to study the closed loop in Figure 3, it is necessary to mix continuous and discrete time signals and systems. This combination of a digital controller and continuous plant is commonly known as hybrid control [Goodwin and Salgado (1994); Feuer and Goodwin (1996); Goodwin et al. (2001)].

We introduce the operator $[S(j \omega)]_{q}$ to denote the folded version of $S(j \omega)$ (Oppenheim and Schafer (1989)), i.e.,

$$
[S(j \omega)]_{q}=\sum_{k=-\infty}^{\infty} S\left(j \omega+j \frac{2 \pi}{\Delta} k\right)
$$

where $S(j \omega)$ can be, either, the Fourier transform of a continuous-time signal, or the Fourier transform of the impulse response of a continuous-time linear system.

We next present three well known results that can be used to analyse the closed loop in Figure 3. We assume a zeroorder hold for simplicity.

Fact 1. The continuous and discrete time signals in Figure 2 satisfy the following identity:

$$
U_{c}(j \omega)=H(j \omega) U\left(e^{j \omega \Delta}\right)
$$




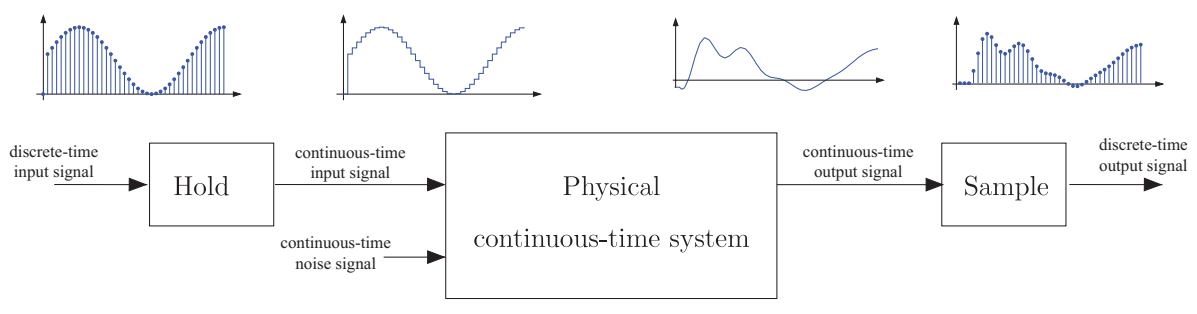

Fig. 1. Sampled system.

where $H(s)=\frac{1-e^{-s \Delta}}{s \Delta}$ is the transfer function of a zero order hold, and $U_{c}(j \omega)=\int_{-\infty}^{\infty} u_{c}(t) e^{-j \omega t} d t$ and $U\left(e^{j \omega \Delta}\right)=$ $\Delta \sum_{k=-\infty}^{\infty} u_{k} e^{-j \omega k \Delta}$ are the continuous-time and (scaled) discrete-time Fourier transforms of $u_{c}(t)$ and $u_{k}$ respectively.

Proof. See e.g. Feuer and Goodwin (1996).

Fact 2. The Fourier transform of the discrete time signals in Figure 2 satisfy the following identity:

$$
Y\left(e^{j \omega \Delta}\right)=[F H]_{q} U\left(e^{j \omega \Delta}\right)
$$

where $H(s)=\frac{1-e^{-s \Delta}}{s \Delta}$ is a zero-order hold, and $Y\left(e^{j \omega \Delta}\right)$, and $U\left(e^{j \omega \Delta}\right)$ are the (scaled) discrete time Fourier transforms given by $Y\left(e^{j \omega \Delta}\right)=\Delta \sum_{k=-\infty}^{\infty} y_{k} e^{-j \omega k \Delta}$, and $U\left(e^{j \omega \Delta}\right)=\Delta \sum_{k=-\infty}^{\infty} u_{k} e^{-j \omega k \Delta}$.

Proof. See e.g. Goodwin et al. (2010b).

Fact 3. The continuous and discrete time signals in Figure 2 satisfy the following identity:

$$
Y\left(e^{j \omega \Delta}\right)=\left[F(j \omega) U_{c}(j \omega)\right]_{q}
$$

where $U_{c}(j \omega)=\int_{-\infty}^{\infty} u_{c}(t) e^{-j \omega t} d t$, and $Y\left(e^{j \omega \Delta}\right)=$ $\Delta \sum_{k=-\infty}^{\infty} y_{k} e^{-j \omega k \Delta}$.

Proof. See e.g. Oppenheim and Schafer (1989).

Remark 1. The above facts can be readily extended to any hold device with Kronecker delta response $H(s)$ (Feuer and Goodwin (1994); Yuz et al. (2004)). $\nabla \nabla \nabla$

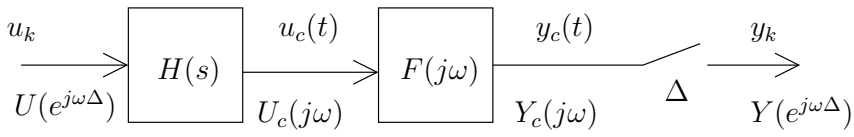

Fig. 2. Signals in a sampling process.

\section{A 2-DOF-AAF}

We next analyse the signals in the closed loop of Figure 3. Proposition 4. The Fourier transform of the input and output of the plant in Figure 3 satisfy the following identities:

$$
\begin{aligned}
U= & \frac{H}{1+C_{q}\left[\left(F_{2} G+F_{1}\right) H\right]_{q}} \\
& \cdot\left\{C_{q}\left(R_{k}-\left[F_{2}\left(D_{m}+D_{0}\right)\right]_{q}\right)+D_{i}\right\} \\
Y= & D_{0}+\frac{G H}{1+C_{q}\left[\left(F_{2} G+F_{1}\right) H\right]_{q}} \\
& \cdot\left\{C_{q}\left(R_{k}-\left[F_{2}\left(D_{m}+D_{0}\right)\right]_{q}\right)+D_{i}\right\}
\end{aligned}
$$

Proof. Using facts 1 and 2 we have that

$$
\begin{gathered}
Y=D_{0}+G U \\
U=H\left(D_{i}+U^{d}\right) \\
U^{d}=C_{q}\left\{R-\left(\left[F_{2}\left(D_{m}+D_{0}\right)\right]_{q}\right.\right. \\
\left.\left.+\left[F_{2}\left(G H\left(D_{i}+U^{d}\right)\right)\right]_{q}+\left[F_{1} H\left(D_{i}+U^{d}\right)\right]\right)\right\}
\end{gathered}
$$

Then, solving for $U^{d}$ in (9), and using (7) and (8) we obtain the result.

In Goodwin et al. (2009) the filters $F_{1}$, and $F_{2}$ are chosen such that the following condition holds:

$$
F_{1}+F_{2} G=G
$$

The latter condition on the filters $F_{1}$ and $F_{2}$ appears in the literature as a tool to design unbiased estimates (Serón et al. (1997)). A general procedure to design $F_{1}$ and $F_{2}$ for a given plant $G$ is presented in the Appendix.

Remark 2. Notice that, when one uses a 2-DOF technique where the filters satisfy (10), it is not necessary to consider the effect of anti-aliasing filters to design the controller. $\nabla \nabla \nabla$

Remark 3. Since anti-aliasing filters are not ideal, aliasing occurs (specially close to the Nyquist frequency). Moreover, the tradeoff between avoiding folding and affecting the signal path suggests the use of a low order anti-aliasing filter. On the other hand, it is possible to use a higher order anti-aliasing filter in the 2-DOF AAF architecture without affecting the signal path. This is potentially of importance in wide-band control. $\nabla \nabla \nabla$

\section{EQUIVALENCE BETWEEN 1-DOF-AAF AND 2-DOF-AAF ARCHITECTURES}

In this section we analyse the potential equivalence between the 2-DOF-AAF and the usual 1-DOF-AAF when the system is operating in closed loop.

We first define when two control loop architectures are considered equivalent. 


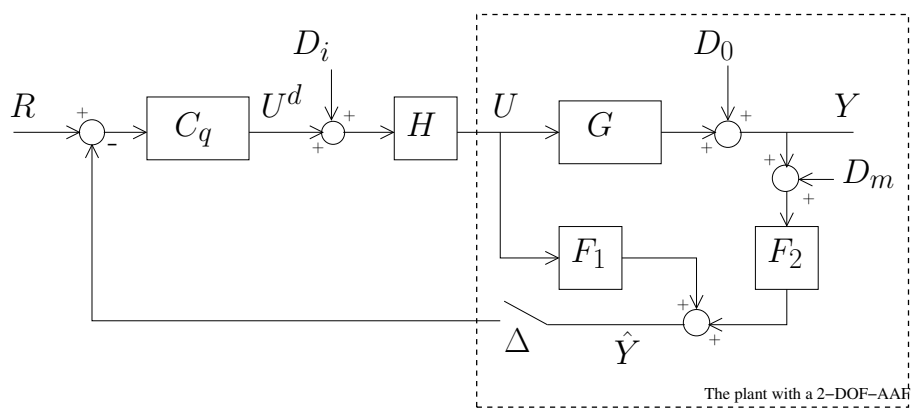

Fig. 3. Closed Loop with 2 degree of freedom anti-aliasing filter.

Definition 1. Two control architectures are equivalent if and only if, for given disturbances $D_{i}, D_{0}, D_{m}$, and any reference signal $R$ (see Figure 3 ), the Fourier transform of the continuous time input and output of the plant are the same for all frequencies. $\nabla \nabla \nabla$

\section{We then have}

Theorem 5. For the control architecture (shown in Figure 3 ) using a 2-DOF-AAF, where the controller is linear, we have that:

(1) If the input disturbance $D_{i}$ is identically zero $\left(D_{i}\left(e^{j \omega \Delta}\right)=0, \forall \omega \in\left[-\frac{\pi}{\Delta}, \frac{\pi}{\Delta}\right]\right)$, then there exists a 1-DOF-AAF control architecture $\left(F_{1}=0\right)$ such that both the 1-DOF and 2-DOF AAF are equivalent. A particular case satisfying the above condition occurs when the controller in the 1-DOF-AAF control is given by:

$$
C_{q}^{\prime}=\frac{C_{q}}{1+C_{q}\left[F_{1} H\right]_{q}}
$$

and the $\mathrm{AAF}$ in the $1-\mathrm{DOF}$ is equal to the filter $F_{2}$ used in the 2-DOF-AAF control architecture.

(2) If the input disturbance $D_{i}$ is not identically zero (i.e. there exists a $\omega_{0} \in\left[-\frac{\pi}{\Delta}, \frac{\pi}{\Delta}\right]$ such that $D_{i}\left(e^{j \omega_{0} \Delta}\right) \neq$ $0)$, then there does not exist a 1-DOF-AAF control architecture that is equivalent to the 2-DOF-AAF architecture.

\section{Proof.}

(1) If $D_{i}$ is identically equal to zero, and the controller is linear, then using Proposition 4 we have that the plant input is given by:

$$
\begin{aligned}
U & =\frac{H C_{q}}{1+C q\left[H\left(F_{1}+F_{2} G\right)\right]_{q}} Z \\
Z & =R-\left[F_{2}\left(D_{m}+D_{0}\right)\right]_{q}
\end{aligned}
$$

then, considering the relationship (11), we have that

$$
\frac{H C_{q}}{1+C_{q}\left[\left(F_{2} G+F_{1}\right) H\right]_{q}}=\frac{H C_{q}^{\prime}}{1+C_{q}^{\prime}\left[F_{2} G H\right]_{q}}
$$

Then, noting that $Z$, in (13), only depends on the filter $F_{2}$, we obtain the result.

(2) In this case we have that the input signal is given by

$$
\begin{gathered}
U=\frac{H}{1+C_{q}\left[H\left(F_{1}+F_{2} G\right)\right]_{q}}\left[C_{q} Z+D_{i}\right] \\
Z=R-\left[F_{2}\left(D_{m}+D_{0}\right)\right]_{q}
\end{gathered}
$$

We use contradiction. We consider a 2-DOF-AAF architecture with $F_{1} \neq 0, F_{2} \neq 0$, and 1-DOF-AAF given by $F_{2}^{\prime}$, and the corresponding controller $C_{q}^{\prime}$. We have that the two control architectures are equivalent if and only if

$$
\begin{aligned}
\frac{H C q Z}{1+C q\left[H\left(F_{1}+F_{2} G\right)\right]_{q}} & =\frac{H C^{\prime} q Z^{\prime}}{1+C q\left[H\left(F_{2}^{\prime} G\right)\right]_{q}} \\
\frac{H}{1+C q\left[H\left(F_{1}+F_{2} G\right)\right]_{q}} & =\frac{H}{1+C q\left[H\left(F_{2}^{\prime} G\right)\right]_{q}}
\end{aligned}
$$

Then, using (18) in (17) we have that $Z C_{q}=C_{q}^{\prime} Z^{\prime}$ where $Z^{\prime}=R-\left[F_{2}^{\prime}\left(D_{m}+D_{0}\right)\right]_{q}$. Then, considering the part of $Z$ and $Z^{\prime}$ that depends only on the reference signal, we have that $C_{q}=C_{q}^{\prime}$. Then, considering only the part of $Z$ and $Z^{\prime}$ that depends on the disturbance signals, we have that $\left[F_{2}\left(D_{m}+D_{0}\right)\right]_{q}=$ $\left[F_{2}^{\prime}\left(D_{m}+D_{0}\right)\right]_{q}$. However, then using (18) we have that $F_{1}=0$. This contradicts the initial assumption, and completes the proof. $\nabla \nabla \nabla$

\section{IMPLICATIONS FOR NETWORKED CONTROL SYSTEMS}

Standard control theory (see e.g. Goodwin et al. (2001)) assumes perfect communication between plant and controllers. However, advances in communication technology have motivated the use of general purpose communication networks in control (see e.g. Nair et al. (2007); Schenato et al. (2007); Hespanha et al. (2007); Goodwin et al. (2008); Silva et al. (2010)). Figure 4 shows a Networked Control System (NCS) where the input commands and the measurements used by the controller are transmitted through a network.

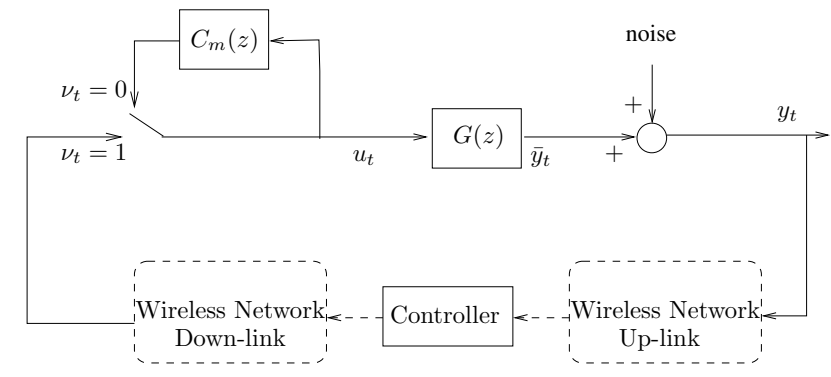

Fig. 4. Networked Control System architecture.

In an NCS framework, new challenges arise. For example, typical communication links are subject to data-rate limits, are prone to data loss, and may experience random delays. In Silva et al. (2010), a simple approach has been studied where the wireless network is replaced by an additive noise source where the associated variance appears as a degree of freedom in the design. 
Hence, in the case that one is designing a controller for a continuous-time plant in an NCS framework it is relevant to consider the case $D_{i} \neq 0$. In this situation, the proposed 2-DOF-AAF cannot be transformed to the usual 1-DOFAAF architecture (see Theorem 5).

\section{NUMERICAL EXAMPLE}

In this section we present a simple example to illustrate the advantages of using the 2-DOF-AAF presented in this paper. Consider the following plant:

$$
G(s)=\frac{1}{s+1}
$$

We use the control architecture of Figure 3, with a zeroorder-hold $(\mathrm{ZOH})$. The sampling frequency is chosen as $\omega_{s}=20[\mathrm{rad} / \mathrm{s}]$ which corresponds to a Nyquist frequency $\omega_{N}=10[\mathrm{rad} / \mathrm{s}]$. We study the following scenarios:

Scenario I The controller is linear and all disturbances and noise are zero, i.e. $d_{i}(k \Delta)=0, d_{0}(t)=0$, and $d_{m}(t)=0$.

Scenario II The controller is linear and the disturbances and noise are Gaussian sequences having zero mean and variance 0.0001 for both $d_{i}(k \Delta)$, and $d_{0}(t)$, and variance 0.05 for $d_{m}(t)$.

Scenario III The controller is linear and we consider that data loss occurs. We consider a sinusoidal output disturbance with frequency $2.3 * \omega_{N}$ and amplitude $1 / 3$. We use the data loss process described in Figure 4 where the data loss process in the measurement, $\nu_{t}$, satisfies a Bernoulli distribution with probability $p=0.1171$.

Scenario IV We use a non-linear MPC controller where all disturbances and noise are zero, i.e. $d_{i}(k \Delta)=0$, $d_{0}(t)=0$, and $d_{m}(t)=0$.

Scenario V We use a non-linear MPC controller where the disturbances and noise are Gaussian sequences having zero mean and variance 0.0001 for both $d_{i}(k \Delta)$, and $d_{0}(t)$, and variance 0.05 for $d_{m}(t)$.

Scenario VI We use a nonlinear MPC controller and the output disturbance is a sinusoidal signal with frequency $2.3 * \omega_{N}$ and amplitude $1 / 3$. We use the data loss process in Figure 4 where the data loss process, $\nu_{t}$, satisfies a Bernoulli distribution with probability $p=0.1171$.

The actuator uses a hold-input strategy to compensate the data loss, i.e., in Figure $4 C_{m}(z)=z^{-1}$ (Schenato et al. (2007))

For scenarios I, II, and III, we design a linear controller for the discretized plant $G_{d}(z)$ using the command lqg of MATLAB, with weighting matrices $Q_{x u}=10^{-5} I_{2}$, $Q_{w v}=\operatorname{diag}(0.0001,0.0001,0.05), Q_{i}=1$. This controller is denoted as $C_{q}(z)$.

For scenarios IV, V, and VI, we design an MPC controller by using the command mpc $(\mathrm{Gd}, \mathrm{h})$ where $h$ is the sampling rate. The weights that we use are: $10^{-5}$ for the control signal rate, and the default values of the command for the rest, i.e., 0 for the magnitude of the control signal, and 1 for the output. We use the following constraint in the control signal: $-1<u_{k}$.

For the 1-DOF-AAF we use a first order Butterworth filter, $B_{1}(s)$, with cut-off frequency $\omega_{c}=\omega_{N}$. We notice that the loop becomes unstable if higher order AAFs are used.

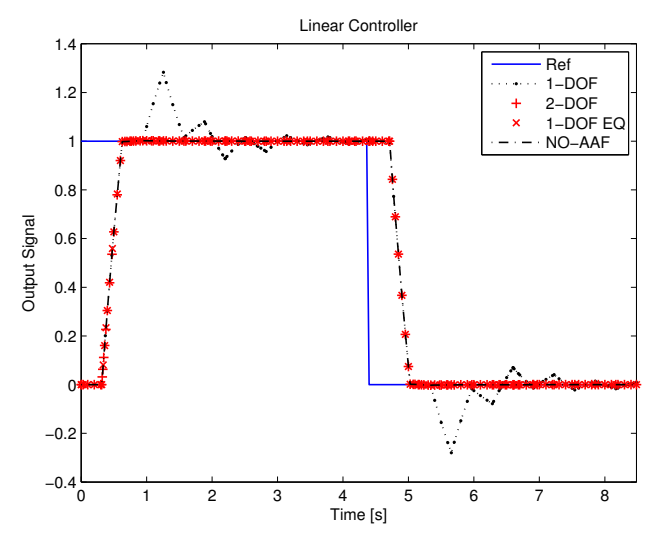

Fig. 5. Simulation for scenario I (Linear controller, Noise free, and without data loss).

For the 2-DOF-AAF we use a fifth order Butterworth filter, $B_{5}(s)$, with cut-off frequency $\omega_{c}=\omega_{N}$ for $F_{2}$ and we choose $F_{1}$ to satisfy (10). For every scenario we show the impact of the following AAF configurations:

NO-AAF: No AAF is used, i.e. $F_{1}(s)=0$, and $F_{2}(s)=1$. 1-DOF: $F_{1}(s)=0$, and $F_{2}(s)=B_{1}(s)$.

2-DOF: We consider $F_{1}(s)$ such that $F_{1}(s)+G(s) F_{2}(s)=$ $G(s)$, and $F_{2}(s)=B_{5}(s)$.

1-DOF EQ: $F_{1}(s)=0, F_{2}(s)=B_{5}(s)$, and we use a controller $C_{q}^{\prime}$ that satisfies (11).

Remark 4. Notice that MPC is typically used in NCS framework (see, e.g. Yu et al. (2004); Liu et al. (2006); Jing et al. (2007); Chen et al. (2010); Pin et al. (2009)). In all the above papers, no anti-aliasing filter is considered in the design of the control system. $\nabla \nabla \nabla$

Table 1 shows the value of the ratio $\frac{\rho}{\rho_{2 D O F}}$, where $\rho=$ $\frac{1}{T} \int_{0}^{T}|z(t)-y(t)| d t, \rho_{2 D O F}$ is the value of $\rho$ for the 2DOF case, $z(t)$ is the reference signal passed through a $\mathrm{ZOH}$, and $T=2638[s]$ is the simulation time.

Figure 5 shows the results for all AAF configurations for scenario I. We see that 2-DOF , 1-DOF EQ, and NO$\mathbf{A A F}$ cases provide good performance. However, for the 1-DOF case, the control loop shows poor performance.

The simulation results for scenario II for all the AAF cases are shown in Figure 6. The 2-DOF and 1-DOF EQ have the same performance (see Table 1). The NO-AAF case has poor performance compared to the $\mathbf{2 - D O F}$ and $\mathbf{1 -}$ DOF case. The $\mathbf{2 - D O F}$ case shows the best performance.

Figure 7 shows the results for scenario III. We see that the 2-DOF case shows the best performance. In Table 1 we notice that, for scenario III, the NO-AAF shows the worst performance.

Figure 8 shows the simulation results for all AAF configurations (except for 1-DOF EQ ), considering scenario IV. The $\mathbf{2 - D O F}$ and NO-AAF cases have the same behaviour (see Table 1). The performance of the $\mathbf{2 - D O F}$ case is better than the performance for the $\mathbf{1 - D O F}$ case.

Simulation results for scenario $\mathbf{V}$ are shown in Figure 9. The performance of the $\mathbf{1 - D O F}$ case is worse than the performance for the NO-AAF. The $\mathbf{2 - D O F}$ gives the best performance. 


\begin{tabular}{|c|c|c|c|c|}
\hline & 1-DOF & 2-DOF & 1-DOF EQ & NO-AAF \\
\hline I (Linear controller, Noise free, and without data loss) & 1.3157 & 1 & 1 & 1 \\
\hline II (Linear controller, with noise, and without data loss) & 1.1313 & 1 & 1 & 1.3033 \\
\hline III (Linear controller, only a sinusoidal disturbance, and with data loss) & 1.3199 & 1 & - & 1.4696 \\
\hline IV (MPC controller, Noise free, and without data loss) & 1.2060 & 1 & - & 1 \\
\hline V (MPC controller, with noise, and without data loss) & 1.0846 & 1 & - & 1.2032 \\
\hline VI (MPC controller, only a sinusoidal disturbance, and with data loss) & 1.1171 & 1 & - & 1.2009 \\
\hline
\end{tabular}

Table 1. Integral of the absolute tracking error over the simulation time ratio, $\frac{\rho}{\rho_{2 D O F}}$, for all the scenarios and AAF configurations.

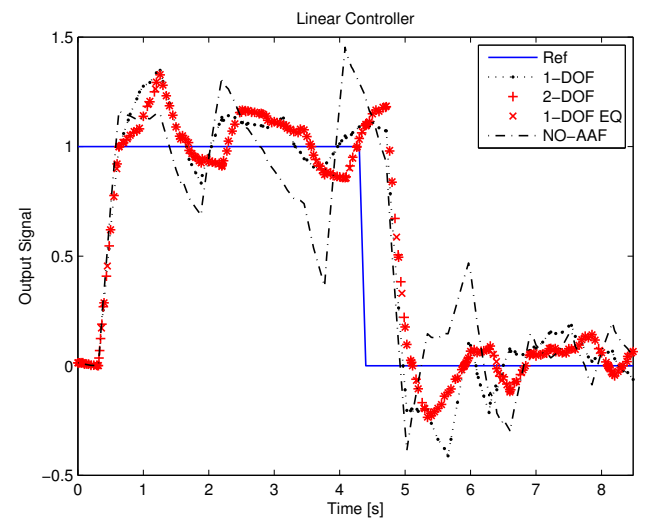

Fig. 6. Simulation for scenario II (Linear controller, with noise, and without data loss).

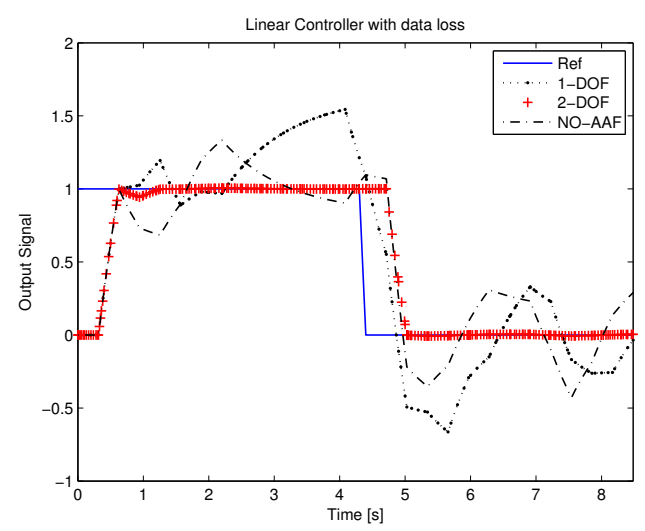

Fig. 7. Simulation for scenario III (Linear controller, only sinusoidal disturbance, and with data loss).

Figure 10 shows the simulation results considering scenario VI. The 2-DOF gives the best performance. Table 1 shows that for scenario VI, the NO-AAF gives the worst performance.

\section{CONCLUSIONS}

In this paper we have studied a new anti-aliasing technique with a two-degree-of-freedom architecture. The new AAF technique reduces the effect of the phase shift without affecting the signal path. This means that the control design procedure becomes independent of sampling artifacts.

We have analysed the potential equivalence between the new $\mathrm{AAF}$ and the usual one-degree-of-freedom antialiasing scheme. We have illustrated the potential of the proposed technique with a simple example using a linear controller and an MPC strategy in an NCS framework.

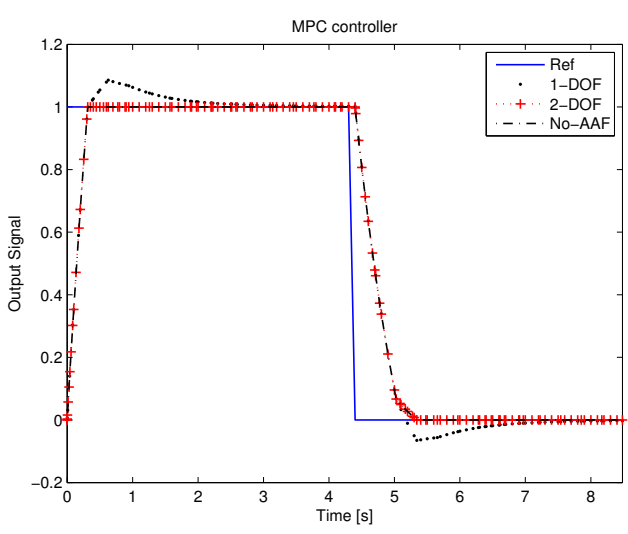

Fig. 8. Simulation for scenario IV (MPC controller, Noise free, and without data loss).

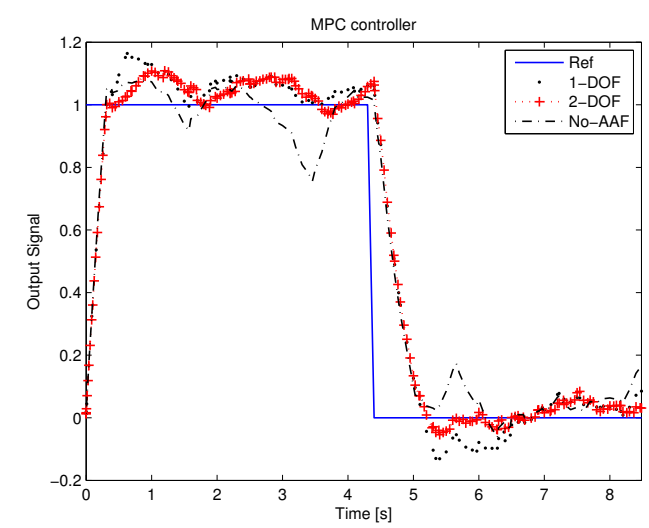

Fig. 9. Simulation for scenario V (MPC controller, with noise, and without data loss).

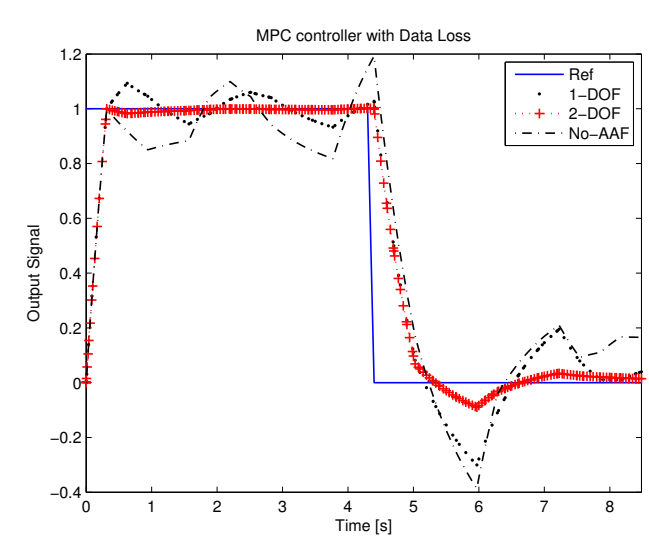

Fig. 10. Simulation for scenario VI (MPC controller, only a sinusoidal disturbance, and with data loss). 


\section{Appendix A. UNBIASED ESTIMATES}

There exists a general class of 2-DOF filters which yield the following key property (see Figure 3): The transfer function from $u(t)$ to $\hat{y}(t)$ is the same as that from $u(t)$ to $y(t)$. This class of filters can be analyzed, in the Fourier transform domain, as follows:

$$
\hat{Y}(j \omega)=F_{1}(j \omega) U(j \omega)+F_{2}(j \omega) Y(j \omega)
$$

Clearly, the transfer function from $u(t)$ to $\hat{y}(t)$ is given by:

$$
\frac{\hat{Y}(j \omega)}{U(j \omega)}=F_{1}(j \omega)+F_{2}(j \omega) G(j \omega)
$$

where $G(j \omega)$ is the plant transfer function. We say that the 2-DOF filter is unbiased if this transfer function is the same as the plant transfer function. Hence, the condition for unbiasedness is:

$$
F_{1}(j \omega)+F_{2}(j \omega) G(j \omega)=G(j \omega)
$$

All linear time-invariant observers have the above property as we show below:

Lemma 6 . Consider a plant represented by the state-space model:

$$
\begin{aligned}
& \dot{x}=A x+B u \\
& y=C x
\end{aligned}
$$

and the class of (full order) observers given by:

$$
\begin{aligned}
& \dot{\hat{x}}=A \hat{x}+B u+J(y-\hat{y}) \\
& \hat{y}=C \hat{x}
\end{aligned}
$$

Then, all such observers are unbiased

Proof. The proof can be found, for example, in Goodwin et al. (2001); Serón et al. (1997)

\section{REFERENCES}

Błachuta, M. and Grygiel, R. (2009a). Are anti-aliasing filters really necessary for sampled-data control? In American Control Conference, 2009. ACC '09., 3200 3205.

Błachuta, M. and Grygiel, R. (2009b). On the effect of anti-aliasing filters on sampled-data PID control. In Control and Decision Conference, 2009. CCDC '09. Chinese, $4904-4909$.

Błachuta, M. and Grygiel, R. (2010). Impact of antialiasing filters on optimal sampled-data PID control. Control and Automation (ICCA), 2010 8th IEEE International Conference on, $1397-1402$.

Chen, J., Irwin, G., and McKernan, A. (2010). Packetbased robust MPC for wireless networked control using co-design. American Control Conference (ACC), 2010, $1829-1834$.

Feuer, A. and Goodwin, G. (1994). Generalized sample hold functions-frequency domain analysis of robustness, sensitivity, and intersample difficulties. Automatic Control, IEEE Transactions on, 39(5), 1042 - 1047.

Feuer, A. and Goodwin, G.C. (1996). Sampling in digital signal processing and control. Birkhauser, Boston.

Goodwin, G.C., Agüero, J.C., and Yuz, J.I. (2009). Two degree of freedom anti-aliasing filter. Patent No 200890608.
Goodwin, G.C., Graebe, S.F., and Salgado, M.E. (2001). Control System Design. Prentice Hall, Upper Saddle River, NJ.

Goodwin, G.C. and Salgado, M.E. (1994). Frequencydomain sensitivity functions for continuous-time systems under sampled-data control. Automatica, 30(8), 1263-1270.

Goodwin, G.C., Yuz, J.I., and Agüero, J.C. (2010a). Models for sampled data systems. In The Control Handbook. CRC Press, 2nd edition.

Goodwin, G.C., Yuz, J.I., Agüero, J.C., and Cea, M. (2010b). Sampling and sampled-data models. American Control Conference (ACC), 2010, $1-20$.

Goodwin, G., Silva, E., and Quevedo, D. (2008). A brief introduction to the analysis and design of networked control systems. In Control and Decision Conference, 2008. CCDC 2008. Chinese, $1-13$.

Hespanha, J., Naghshtabrizi, P., and Xu, Y. (2007). A survey of recent results in networked control systems. Proceedings of the IEEE, 95(1), $138-162$.

Jing, W., Liqian, Z., and Tongwen;, C. (2007). An MPC approach to networked control design. Control Conference, 200\%. CCC 200\%. Chinese, 10-14.

Liu, G.P., Mu, J.X., Rees, D., and Chai, S.C. (2006). Design and stability analysis of networked control systems with random communication time delay using the modified mpc. International Journal of Control, 79(4), $288-297$.

Nair, G., Fagnani, F., Zampieri, S., and Evans, R. (2007). Feedback control under data rate constraints: An overview. Proceedings of the IEEE, 95(1), $108-137$.

Nyquist (1928). Certain topics in telegraph transmission theory. American Institute of Electrical Engineers, Transactions of the, 47(2), $617-644$.

Oppenheim, A.V. and Schafer, R.W. (1989). Discrete-time signal processing. Prentice Hall.

Pin, G., Filippo, M., and Parisini, T. (2009). Networked MPC for constrained linear systems: a recursive feasibility approach. Decision and Control, 2009 held jointly with the 2009 28th Chinese Control Conference. $C D C / C C C$ 2009. Proceedings of the 48th IEEE Conference on, $555-560$.

Schenato, L., Sinopoli, B., Franceschetti, M., Poolla, K., and Sastry, S. (2007). Foundations of control and estimation over lossy networks. Proceedings of the IEEE, 95(1), $163-187$.

Serón, M.M., Braslavsky, J.H., and Goodwin, G.C. (1997). Fundamental Limitations in Filtering and Control. Springer-Verlag.

Silva, E.I., Agüero, J.C., Goodwin, G.C., Lau, K., and Wang, M. (2010). The SNR approach to networked control. In The Control Handbook. CRC Press, 2nd edition.

Yu, J.Y., Mei, Y.M., and Yu, S.M. (2004). MPC-based method dealing with vacant sampling and data rejection in networked control systems. Machine Learning and Cybernetics, 2004. Proceedings of 2004 International Conference on, 1, $574-578$ vol.1.

Yuz, J.I., Goodwin, G.C., and Garnier, H. (2004). Generalised hold functions for fast sampling rates. In ecision and Control, 2004. CDC. 43rd IEEE Conference on (2004), volume 2, 1908-1913. 